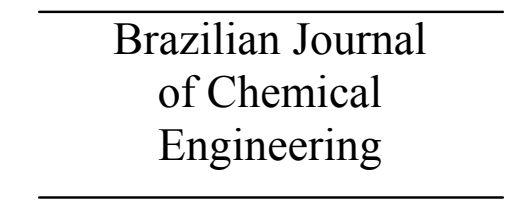

ISSN 0104-6632

Printed in Brazil

www.abeq.org.br/bjche

Vol. 27, No. 04, pp. 619 - 626, October - December, 2010

\title{
PREPARATION OF ZEOLITE A COATINGS ON COPPER PLATES BY USING THE SUBSTRATE HEATING METHOD
}

\author{
S. Teber, M. Elnekave and M. Tatlier ${ }^{*}$ \\ Department of Chemical Engineering, Istanbul Technical University, \\ Phone: + 90212 2856234, Fax: + 90212 2852925, Maslak, 34469 Istanbul, Turkey. \\ E-mail: tatlierm@itu.edu.tr
}

(Submitted: March 27, 2010 ; Revised: July 5, 2010 ; Accepted: July 28, 2010)

\begin{abstract}
The substrate heating method was used to grow zeolite 4A coatings on copper plates. The reaction mixture was kept at $25^{\circ} \mathrm{C}$, while the plate was heated to a higher temperature. In some of the synthesis experiments performed, the reaction mixture was circulated in the system. The coatings prepared were characterized by X-ray diffraction and scanning electron microscopy. It was demonstrated that crystalline and pure zeolite $4 \mathrm{~A}$ coatings could be formed on copper by using the substrate heating method from a highly alkaline solution, in which dissolution and oxidation of the copper plates took place. A coating with about 310 $\mu \mathrm{m}$ mass equivalent thickness could be grown on copper after $72 \mathrm{~h}$ of synthesis. The thickness decreased when circulation was applied. Compared to coatings previously prepared on stainless steel plates under similar experimental conditions, thicker coatings were grown on copper. It is possible that the roughened surface of copper in the highly alkaline reaction mixture provided a higher number of nucleation sites.

Keywords: Zeolite; Coating; Copper; Circulation.
\end{abstract}

\section{INTRODUCTION}

Zeolites are hydrated microporous crystalline materials with unique properties, which enable them to be used in diverse applications, mainly as catalysts, ion exchangers and adsorbents. Support materials coated by zeolites may serve as sensors (Mintova and Bein, 2001), membranes in separation processes (Jareman and Hedlund, 2005), structured catalysts in reactors (Yuranov et al., 2005) and adsorbents in adsorption heat pumps (Tatlier et al., 1999). Zeolite coatings may be prepared by a variety of synthesis procedures (Sterte et al., 1997; Yamazaki and Tsutsumi, 1995; Erdem-Şenatalar et al., 1999), resulting in coatings of different characteristics that may be favorable for use in different applications. Variations occur in the thickness, orientation and homogeneity of the zeolite films. Thin, uniform and continuous zeolite films are required for some specific applications like membrane separations. Orientation of the crystals on the surface may also be important, depending on the pore structure of the zeolite. For some other applications related to adsorption or catalysis, thicker coatings might be required for optimal performance, while continuous and defectfree formation is not very important.

Zeolites are crystalline aluminosilicates that are metastable in nature and they tend to transform into more stable phases after a prolonged time. This may present a serious difficulty for obtaining relatively thick zeolite coatings. This problem might be overcome by using the substrate heating method, which allows a better manipulation of zeolite synthesis by controlling the temperature of the metal substrate, independent from that of the reaction mixture (Erdem-Şenatalar et al., 1999). In this

*To whom correspondence should be addressed 
method, the phase transformations of the metastable zeolites can be delayed for long periods of time, depending on the volume of the reaction mixture, and the growth of zeolite coatings of various thicknesses on metal supports may be achieved. The coatings prepared by the substrate heating method exhibited differences in their nature arising from their growth under a thermal gradient (Erdem-Şenatalar et al., 1999). The inhomogeneities in the coating thickness at earlier times of synthesis resulted finally in an accessible sponge-like structure. By using this method, zeolite coatings of up to about $150 \mu \mathrm{m}$ and $270 \mu \mathrm{m}$ mass equivalent thickness could be prepared on $1 \times 1 \mathrm{~cm}^{2}$ (Erdem-Şenatalar et al., 1999) and $5 \times 5$ $\mathrm{cm}^{2}$ (Tatlier et al., 2007) stainless steel plates, respectively. The mass equivalent thickness is defined as (mass of zeolite deposited per coated area of substrate)/(density of zeolite).

There are numerous reports in the literature on the preparation of zeolite coatings on various substrates. Coatings have been grown on metals, silicon wafers, single crystals and ceramic surfaces (Anderson et al., 1992; Tsay and Chiang, 2000; Mintova and Valtchev, 1996; Valtchev et al., 1997; Scheffler et al., 2004; Beving et al., 2008). Owing to the remarkably stable nature of stainless steel even in highly alkaline and corrosive environments, this metal has generally been selected as the support material for zeolite coatings. A passive film consisting of chromium oxide and hydroxide covers the stainless steel plate, which prevents surface corrosion and blocks corrosion from spreading into the metal's internal structure. The hydroxyl groups of the surface passive film, which play a crucial role in interaction with molecules, are considered to dissociate, depending on the $\mathrm{pH}$ value of the contacting solution (Suzuki, 2006). When the $\mathrm{pH}$ is lower than the iep (isoelectric point, which is the $\mathrm{pH}$ at which a particular molecule or surface carries no net electrical charge) of the metal oxide surface, the hydroxyl groups are in a state of $-\mathrm{OH}_{2}{ }^{+}$. When the $\mathrm{pH}$ is higher than the iep of the metal oxide surface, the hydroxyl groups change to $-\mathrm{O}^{-}$. It is commonly believed that the zeolite is somehow bonded to the oxygen on the surface of stainless steel.

The utilization of zeolite coatings directly grown on copper may be very advantageous for some applications, such as adsorption heat pumps that require fast heating and cooling of the adsorbent. The high thermal conductivity of copper may provide a means to decrease the cycle durations of adsorption heat pumps, thus improving their performance. Regarding the growth of zeolite coatings on copper, experimental conditions, especially the $\mathrm{pH}$ of the reaction mixture, may be very significant in the realization of this process. Adverse effects of alkaline reaction mixtures on the substrate and, consequently, unfavorable effects of the dissolution of copper on the mixture may be possible.

The formation of zeolite Y, silicalite-1 and SAPO-5 from gel mixtures on untreated and deformed copper was investigated (Mintova et al., 1996; Valtchev et al., 1994). The reaction mixture used to produce zeolite $Y$ had the highest $\mathrm{pH}$ value among the three, while that forming SAPO-5 was acidic in nature. Copper partially dissolved and became less stable in the presence of the higher alkalinity, but this seemed to improve the adhesivity of the zeolite $\mathrm{Y}$ coating (Mintova et al., 1996). The plastic deformation of the substrate also led to a similar effect (Valtchev et al., 1994). SAPO-5 coatings on copper, on the other hand, adhered only slightly to the surface (Mintova et al., 1996). In another study, MFI-type zeolite coatings were grown on copper wire gauzes from different reaction mixture compositions (Brehm and Zanter, 2002). The stabilities of the MFI coatings prepared on copper varied with the experimental conditions used. Finally, zeolite $4 \mathrm{~A}$ was grown on specially prepared open-cell copper foam by a two-step synthesis (Bonaccorsi et al., 2006). The copper foam was first seeded by a colloidal seed solution, after which hydrothermal synthesis was applied. The copper foam surface could be coated with a compact layer of zeolite $4 \mathrm{~A}$ firmly bonded to the substrate. A black coating of copper oxide was reported to form on the surface of the copper foam during the seeding treatment, which acted as an interlayer between the metal surface and the growing zeolite crystals. The zeolite coating was estimated to be about $10 \mu \mathrm{m}$ in thickness. Although there are a few studies in the literature involving the growth of zeolites on copper substrates, the possible effects of zeolite synthesis conditions on copper have not been explored in much detail. This may be important since the preparation of zeolite coatings on copper does not seem to be a straightforward task to perform at all due to the possible dissolution and oxidation of copper in the synthesis mixture.

In this study, the formation of zeolite $4 \mathrm{~A}$ on copper plates was investigated by using the substrate heating method, which may permit the preparation of relatively thick coatings. The effects of the reaction mixture on copper were also observed. Some of the experiments were performed by circulating the reaction mixture in the system. The coatings prepared with different thicknesses were characterized by X-ray diffraction (XRD) and scanning electron microscopy (SEM). The results were then compared to those obtained previously by using stainless steel plates. 


\section{EXPERIMENTAL}

Zeolite 4A coatings were prepared on electrolithic copper plates of $1 \times 1 \mathrm{~cm}^{2}$ surface area. The copper content of the plates was $99.9 \%$ and their thickness was equal to $1 \mathrm{~mm}$. The synthesis experiments were performed by using the substrate heating method (Erdem-Şenatalar et al., 1999). The substrate heating device consisted of a polypropylene vessel $(500 \mathrm{ml})$, a heating resistance $(30 \mathrm{~W})$ and a stainless steel rod covered with Teflon. The resistance inserted into the rod was utilized to heat preferentially the side of the metal rod where the copper plate was placed vertically. The system was immersed into the reaction mixture in the polypropylene vessel. The temperature was measured by a thermocouple placed between the metal rod and the heating resistance.

The synthesis experiments were carried out in a water bath kept at $25^{\circ} \mathrm{C}$, while a resistance temperature of $140^{\circ} \mathrm{C}$ was utilized. The synthesis duration was varied between 7 and $72 \mathrm{~h}$. Under these conditions, the surface temperature of the substrate was estimated to be around $57^{\circ} \mathrm{C}$ (Erdem-Şenatalar et al., 1999). A highly alkaline clear solution composition equivalent to $50 \mathrm{Na}_{2} \mathrm{O}: \mathrm{Al}_{2} \mathrm{O}_{3}: 5 \mathrm{SiO}_{2}$ : $1000 \mathrm{H}_{2} \mathrm{O}$ was used in the experiments. Prior to the synthesis of the zeolite coatings, the copper plates were cleaned by rinsing with ethanol and then deionized water. Granular sodium aluminate (Riedelde Haen), anhydrous $\mathrm{NaOH}$ pellets (Carlo Erba), sodium silicate solution (Merck) and deionized water were used in the synthesis experiments. After the synthesis was completed, the system was cooled immediately and the copper plates were removed from the device and washed with deionized water.

In some of the experiments performed, the synthesis mixture was circulated in the batch system, consisting

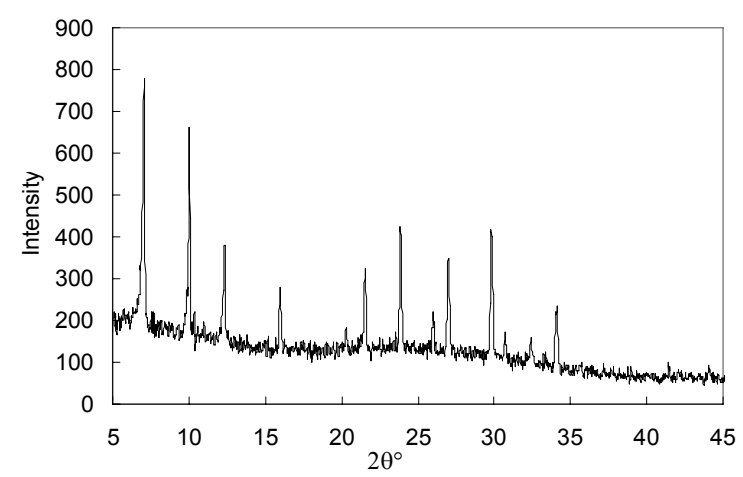

Figure 1: X-ray diffractogram of the sample obtained after $48 \mathrm{~h}$ of synthesis under static conditions. of the reactor and a polypropylene tube, by means of a peristaltic pump. The flow rate of the reaction mixture was equal to $130 \mathrm{ml} / \mathrm{min}$. These experiments were performed to obtain further variations in the coating thickness. X-ray diffraction (Pananalytical X'Pert Pro) was used to characterize the samples synthesized on copper substrates. XRD was run at an incidence angle of $1^{\circ}$ with $\mathrm{Cu} \mathrm{K} \mathrm{K}_{\alpha} 1$ radiation and was applied for phase identification and determination of the purity of the samples. Scanning electron microscopy (JEOL 5410) was used to investigate the quality of some of the coatings prepared

\section{RESULTS AND DISCUSSION}

Coatings were prepared on copper plates at water bath and heating resistance temperatures of $25^{\circ} \mathrm{C}$ and $140^{\circ} \mathrm{C}$, respectively, by using the substrate heating method. XRD analyses were performed to identify the crystalline phase(s) in the coatings. The results indicated that zeolite A coatings could be obtained under both static and circulating conditions for all the synthesis times investigated. Utilizing copper instead of stainless steel plates did not seem to affect the type of the crystalline phase formed on the metal from the highly alkaline reaction mixture used in this study.

The X-ray diffractograms of the samples prepared after $48 \mathrm{~h}$ and $72 \mathrm{~h}$ of syntheses under static conditions may be seen in Figure 1 and Figure 2, respectively. The XRD peaks corresponded to zeolite $A$ and no other phases were observed on copper even after 3 days of synthesis. These results were similar to those obtained in a previous study dealing with the preparation of zeolite 4A coatings on stainless steel plates by using similar experimental conditions (Erdem-Şenatalar et al., 1999).

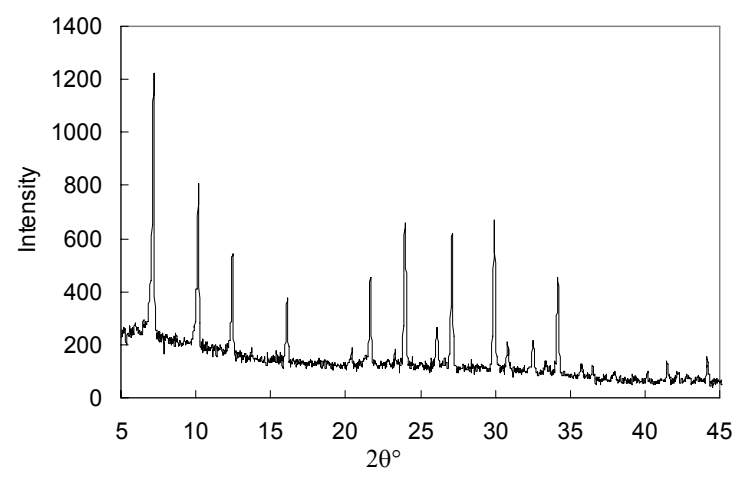

Figure 2: X-ray diffractogram of the sample obtained after $72 \mathrm{~h}$ of synthesis under static conditions. 
The SEM picture depicting the nature of the coating grown after a synthesis duration of $7 \mathrm{~h}$ under static conditions is given in Figure 3. There was a structural change of the copper surface, when compared to the uncoated plate. A visible black coating of copper oxide appeared on the surface of the plate. Scratches were formed and an erosion of the surface could be observed. Small and mostly rounded particles (with $<1 \mu \mathrm{m}$ average size) covered the surface of the copper plate. According to previous experience (Erdem-Şenatalar et al., 1999), these particles should be at least partly amorphous. The SEM picture of the coating grown after a synthesis duration of $18 \mathrm{~h}$ is shown in Figure 4. At this stage of synthesis, the visible particles had about 2-3 $\mu \mathrm{m}$ average size and generally still had rounded edges. The zeolite A crystal growth was intense and covered the whole surface. It may be seen from the SEM pictures that, as the synthesis time was extended, variations in the coating thickness were magnified, resulting in a tree-like growth, and intercrystalline voids were formed in the coating. A similar tree-like nature had been observed for the coatings prepared on stainless steel, but starting at much later synthesis times, longer than $48 \mathrm{~h}$ (Erdem-Şenatalar et al., 1999). The SEM picture of the coating prepared after $24 \mathrm{~h}$ of synthesis

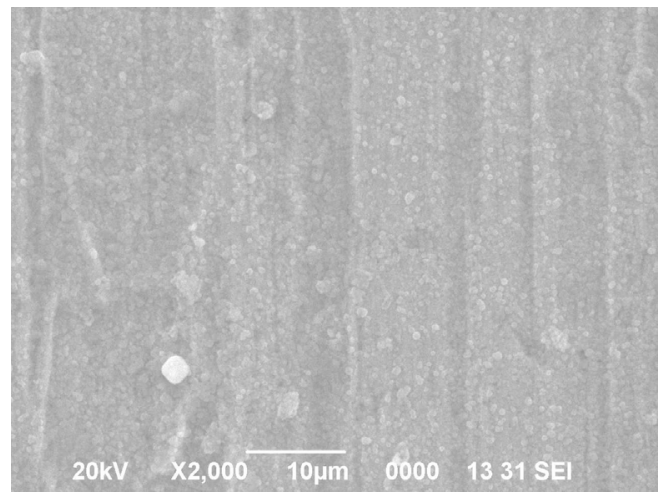

Figure 3: SEM image of the coating obtained after $7 \mathrm{~h}$ of static synthesis. is shown in Figure 5. In this case, where the coating was thicker, the particles on top were about $1 \mu \mathrm{m}$ in size. The crystals were highly intergrown and not loosely attached to the coating surface or to other crystals. A tree-like growth was again apparent from the picture and it should be mentioned that the diversity in the coating thickness amplified with time, leading to such structure. The tree-like growth of the coatings persisted at longer durations, too.

Zeolite A generally exhibits cubic crystal morphology. However, in some studies, rounded particles were observed to form, especially at the beginning of synthesis, after which their edges became sharper (Çetin et al., 2001). This may be related to the presence of some amorphous material in the samples in the early periods of zeolite formation. It is also known that the synthesis conditions may affect the crystal habit. For example, the initially high supersaturation of the reaction mixture has been claimed to lead to spherical crystals (Round et al., 1997). As mentioned above, in this study, the particles obtained at relatively early periods of synthesis were rounded. The crystal edges sharpened to some extent after $24 \mathrm{~h}$, though it is difficult to observe this clearly from Figure 5, due to the presence of highly intergrown small crystals.

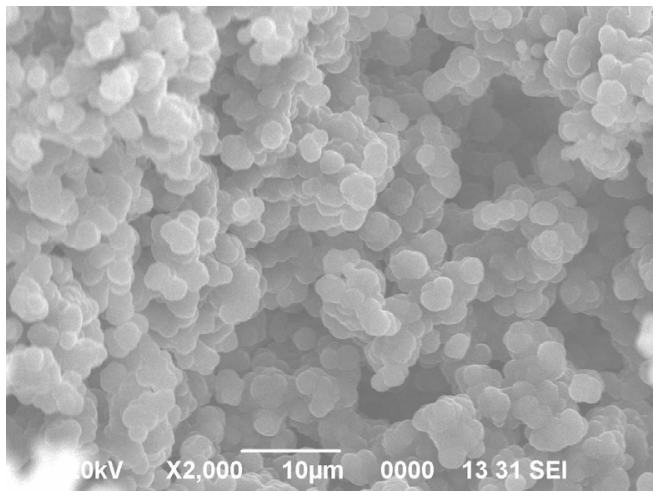

Figure 4: SEM image of the coating obtained after $18 \mathrm{~h}$ of static synthesis.

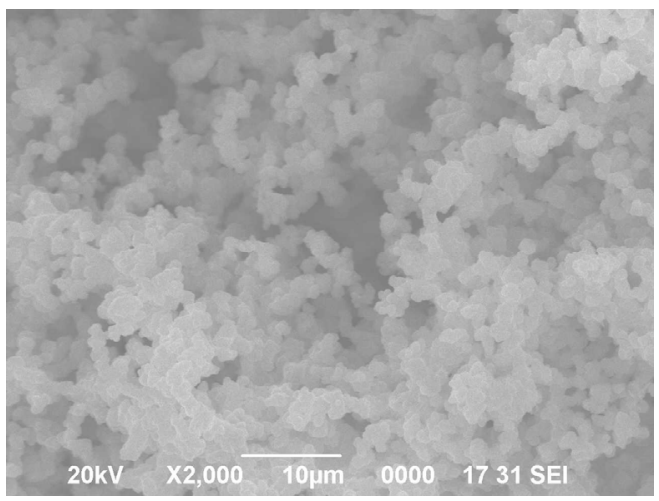

Figure 5: SEM image of the coating obtained after $24 \mathrm{~h}$ of static synthesis. 
In the substrate heating synthesis method, an almost constant nucleation rate may be assumed to exist on the substrate since the concentration of the bulk of the reaction mixture may remain nearly constant for a significantly long period of time. The relatively high significance of nucleation when compared to crystal growth in this method leads to a tree-like growth (Tatlier, 2003). The high nucleation rate is also responsible for the existence of quite small particles, even at relatively long synthesis durations, since crystal growth does not govern the synthesis process.

When circulation was applied in the syntheses, the phase of the zeolite coating obtained on copper plates did not show any variation for any of the durations investigated and pure zeolite A coatings could still be prepared. The X-ray diffractograms of the samples obtained after $48 \mathrm{~h}$ and $72 \mathrm{~h}$ when the reaction mixture was circulated in the synthesis system may be seen in Figure 6 and Figure 7,

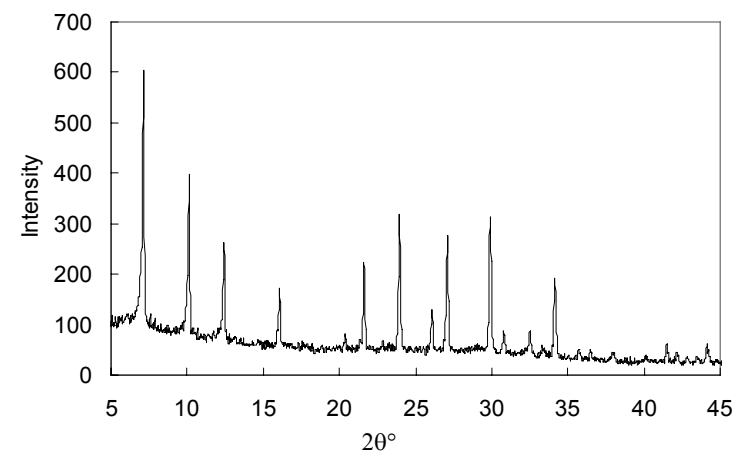

Figure 6: X-ray diffractogram of the sample obtained after $48 \mathrm{~h}$ of synthesis under circulating conditions. respectively. The SEM picture of the coating grown after a synthesis duration of $24 \mathrm{~h}$ under circulation is shown in Figure 8. Similar to the coatings prepared by static synthesis, a tree-like growth with intercrystalline voids was oberved and the zeolite crystals were highly intergrown. The coating mass decreased under circulating conditions. The mass values obtained will be presented later in this section. A similar observation was made in another study where circulation was used to prepare MFI type zeolite membranes on seeded tubular alumina supports (Çulfaz et al., 2006). However, that study involved the use of a flow system instead of a batch one, as employed in this study. The membranes obtained by circulation in the mentioned flow system were reported to be thinner than those synthesized in a static batch system (Çulfaz et al., 2006). This was considered to be related to the decrease in local temperature gradients and composition variations occurring near the metal plate.

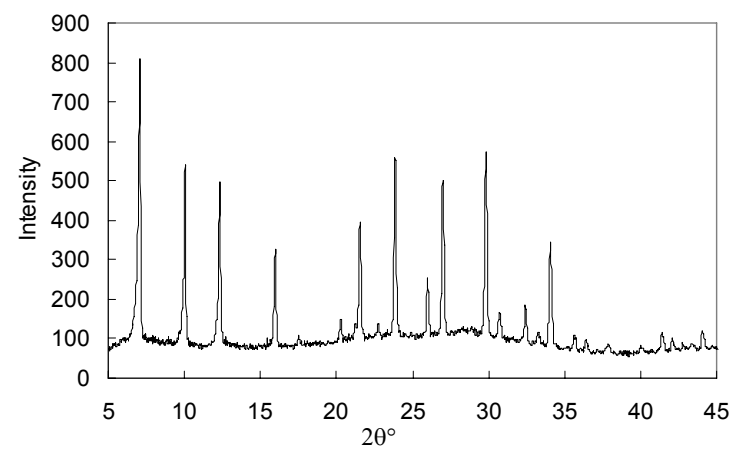

Figure 7: X-ray diffractogram of the sample obtained after $72 \mathrm{~h}$ of synthesis under circulating conditions.

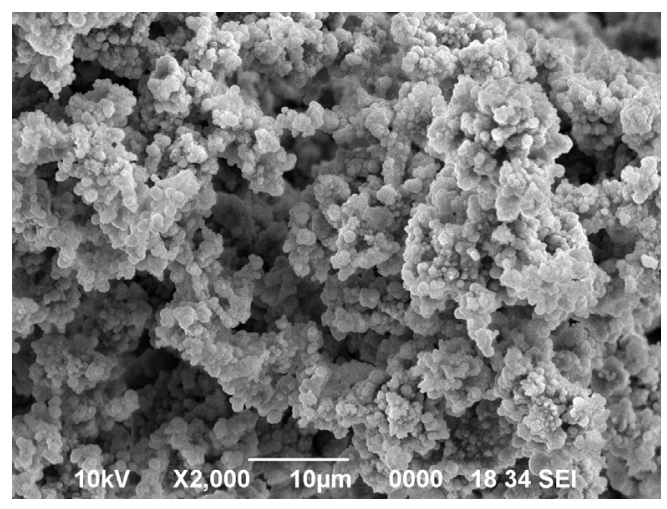

Figure 8: SEM image of the coating obtained after $24 \mathrm{~h}$ of synthesis under circulation. 
The presence of a significant temperature gradient is essential in the substrate heating system, which also leads to enhanced synthesis activity around the metal plate to be coated, resulting in variations in the composition in this region. This concentration gradient may promote the motion of fresh reagents used in zeolite synthesis towards the plate. The utilization of circulating conditions might be expected to reduce the effects of temperature and concentration gradients in the substrate heating system, thus slowing down the rate of nucleation and crystal growth. Actually, notably thinner zeolite A coatings were obtained when the reaction mixture was circulated in the substrate heating system and the nucleation process started much later than that occurring under static conditions. Decreasing the synthesis temperature may also provide thinner coatings, but not in every case, since different zeolites may be formed from the same composition when the temperature is varied. Additionally, the preparation of a thin zeolite coating at a lower temperature may take a considerably longer period of time, when compared to obtaining a coating of the same thickness by the circulation of the reaction mixture at a higher temperature. In other words, the use of circulation may be favorable in the substrate heating system when it is desired to produce relatively thin zeolite coatings.

It was observed that the mass of the copper plates decreased to some extent in the reaction mixture used in this study. The variation of copper mass under different synthesis conditions was investigated. As a first approach, zeolite coatings (discerned from copper oxide by its white color) that were formed on the copper plates under different synthesis conditions were carefully scratched. The plates were then weighed to determine the difference between the original mass of the plate before synthesis and that after the scratching of the zeolite synthesized on the plate. It was determined that copper lost at most about $0.1 \%$ of its original mass during synthesis. As a second approach, a mixture with a $\mathrm{pH}$ similar to the synthesis solution used in this study was prepared. A difference was that sodium silicate was not included in the solution so that zeolite formation would not take place on the copper plates. Copper was treated with this solution at $25^{\circ} \mathrm{C}, 57^{\circ} \mathrm{C}$ (reaction temperature) and $80^{\circ} \mathrm{C}$. From the results depicted in Figure 9, it can be observed that the mass of the copper plates decreased with both increasing time and temperature. At the reaction temperature, the mass decrease amounted to about $0.1 \%$ of the original mass in the most extreme case, in accordance with the first set of investigations.

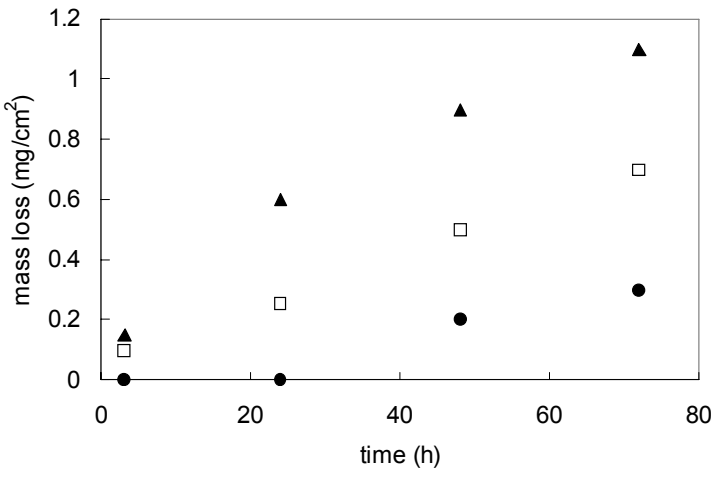

Figure 9: Variation of the mass lost by copper plates with time at $(\bullet) 25^{\circ} \mathrm{C},(\square) 57^{\circ} \mathrm{C}$ and $(\boldsymbol{\Delta}) 80^{\circ} \mathrm{C}$.

When copper is immersed in the reaction mixture, two different phenomena, namely, the dissolution of the plate and the formation of an oxide layer on its surface may be expected to occur. The former and latter phenomena lead to decreases and increases in the mass of the copper plate, respectively. The dissolution of copper seemed to affect the copper mass more significantly in this study, for the conditions investigated. The dissolution of copper might be responsible for the roughened surface of the plates, observed in the SEM pictures. A visible black coating of copper oxide was formed on the plates after the syntheses carried out in the reaction mixture. This might be copper (I) oxide $\left(\mathrm{Cu}_{2} \mathrm{O}\right)$, which forms at relatively low temperatures, below $150^{\circ} \mathrm{C}$.

In order to determine the zeolite coating mass, the measured value of the coating was added to the amount of decrease observed in the mass of the copper plate when treated in the reaction mixture. The variation of coating mass with synthesis duration may be seen in Figure 10 for the different cases investigated. Mass values obtained in a previous study (Erdem-Şenatalar et al., 1999) for stainless steel plates under similar static experimental conditions are also shown in the figure for comparison. Zeolite A coatings of quite different thicknesses were obtained for the different synthesis conditions used. It may be observed from the figure that the amount of zeolite grown on copper by using the substrate heating method increased steadily and almost linearly with synthesis time. The mass equivalent thickness was estimated to be equal to about $310 \mu \mathrm{m}$ for the sample obtained after $72 \mathrm{~h}$ of static synthesis. The actual thickness is higher than the mass equivalent thickness due to the inhomogeneous open textures of these coatings with intercrystalline voids. This type of inhomogeneous coating does not allow the measurement of a single 
actual thickness and only an average value may be determined, which does not provide much information about the coating. Thus, reporting the mass equivalent thickness seems to be the most reasonable approach for the coatings prepared by using the substrate heating method.

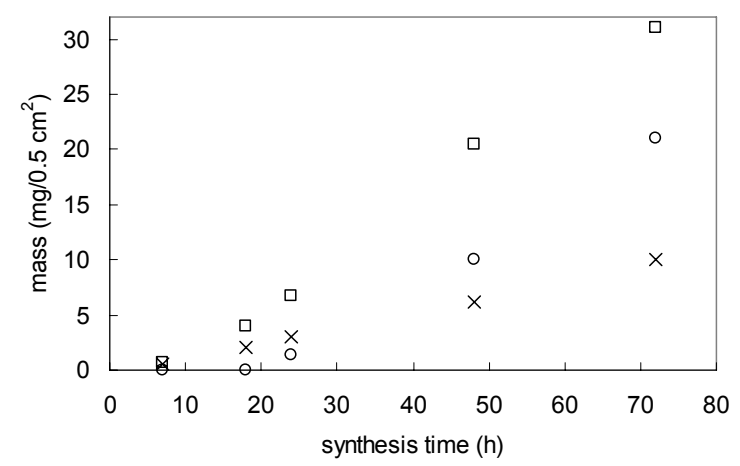

Figure 10: Variation of coating mass with respect to synthesis time for: ( $\square$ ) copper plates and static conditions; (o) copper plates and circulating conditions; and $(\mathrm{x})$ stainless steel plates and static conditions (Erdem-Şenatalar et al., 1999).

When the reaction mixture was circulated, coating formation started at a quite later point in time and, as mentioned before, the mass of the coatings decreased noticeably for all the cases investigated. This decrease was equal to about $50 \%$ and $33 \%$ for the samples prepared after $48 \mathrm{~h}$ and $72 \mathrm{~h}$ of synthesis, respectively. The latter sample had a mass equivalent thickness of about $210 \mu \mathrm{m}$.

Utilizing copper instead of stainless steel led to significant increases in the coating mass under static synthesis conditions. The largest difference was observed for the synthesis carried out for $72 \mathrm{~h}$, for which a three-fold increase was obtained. It seems that the roughened surface of the copper plate promoted the attachment of zeolite/amorphous particles. The copper oxide film that formed on the plate, as mentioned before, probably facilitated the bonding of the zeolite particles to the copper surface. The possible increase in the number of nucleation sites due to the roughened surface of the copper plate might explain the increase in the coating mass obtained for this metal.

\section{CONCLUSIONS}

Crystalline and pure zeolite 4A coatings could be prepared on copper plates by using the susbstrate heating method. The mass of the zeolite increased with synthesis time. Copper did not seem to affect adversely the formation of zeolite coatings from the highly alkaline reaction mixture used in this study, though the mass of the metal plate decreased by about $0.1 \%$. The formation of copper oxide on the surface of the plate, as well as the roughened surface of the support due to its dissolution, seemed to promote the attachment of the zeolite particles by providing more favorable and higher number of sites for zeolite nucleation and crystallization. This resulted in the formation of thicker zeolite A coatings on copper when compared to those previously grown on stainless steel. An increase of about 3-fold was observed after $72 \mathrm{~h}$ of static synthesis. When circulation was applied, pure zeolite A coatings could still be prepared on copper. The coating thickness decreased under circulating conditions, which might be related to a significant decrease in the local temperature and concentration gradients present near the metal plate in the substrate heating system. This type of synthesis might be favorable when it is desired to prepare relatively thin zeolite coatings.

The thickness of coatings grown on copper, obtained by static synthesis, can be further increased in the substrate heating method by varying the synthesis time and water bath temperature since the transformation of zeolite A to another phase should only occur after a much longer period of time. Due to the high thermal conductivity of copper, a zeolitecopper integrated system may be very useful in some applications where fast heating and cooling of the adsorbent is required. In particular, this kind of system is expected to increase further the performance of adsorption heat pumps.

It should be remembered that the significant effects observed in this study of temperature and time on the variation of the mass of copper treated with the reaction mixture implies that unexpected results might be obtained in the preparation of zeolite coatings on copper under quite different synthesis conditions.

\section{REFERENCES}

Anderson, M. W., Pachis, K. S., Shi, J. and Carr, S. W., Synthesis of Self-Supporting Zeolite Films. Journal of Materials Chemistry, 2, 255 (1992).

Beving, D. E., O'Neill, C. R. and Yan, Y., Hydrophilic and Antimicrobial Low-SilicaZeolite LTA and High-Silica-Zeolite MFI Hybrid Coatings on Aluminum Alloys. Microporous and Mesoporous Materials, 108, 77 (2008). 
Bonaccorsi, L., Freni, A., Proverbio, E., Restuccia, G. and Russo, F., Zeolite Coated Copper Foams for Heat Pumping Applications. Microporous and Mesoporous Materials, 91, 7 (2006).

Brehm, A. and Zanter, K.-D., Formation of Zeolite (MFI) Layers on Gauze Wire and Arranged Packing Segment. Chemical Engineering \& Technology, 25, 917 (2002).

Çetin, T., Tatlier, M., Erdem-Şenatalar, A., Demirler, U. and Ürgen, M., Lower Temperatures for the Preparation of Thinner Zeolite A Coatings. Microporous and Mesoporous Materials, 47, 1 (2001).

Çulfaz, P. Z., Çulfaz, A. and Kalıpçılar, H., Preparation of MFI Type Zeolite Membranes in a Flow System with Circulation of the Synthesis Solution. Microporous and Mesoporous Materials, 92, 134 (2006).

Erdem-Şenatalar, A., Tatlier, M. and Ürgen, M., Preparation of Zeolite Coatings by Direct Heating of the Substrates. Microporous and Mesoporous Materials, 32, 331 (1999).

Jareman, F. and Hedlund, J., Single Gas Permeance Ratios in MFI Membranes: Effects of Material Properties and Experimental Conditions. Microporous and Mesoporous Materials, 82, 201 (2005).

Mintova, S., Valtchev, V. and Konstantinov, L., Adhesivity of Molecular Sieve Films on Metal Substrates. Zeolites, 17, 562 (1996).

Mintova, S. and Valtchev, V., Deposition of Zeolite A on Vegetable Fibers. Zeolites 16, 31 (1996).

Mintova, S. and Bein, T., Nanosized Zeolite Films for Vapor-Sensing Applications. Microporous and Mesoporous Materials, 50, 159 (2001).

Round, C. I., Hill, S. J., Latham, K. and Williams, C. D., The Crystal Morphology of Zeolite A. The Effects of the Source of the Reagents. Microporous Materials, 11, 213 (1997).

Scheffler, F., Herrmann, R., Schwieger, W. and Scheffler, M., Preparation and Properties of an Electrically Heatable Aluminium Foam/Zeolite
Composite. Microporous and Mesoporous Materials, 67,53 (2004).

Sterte, J., Mintova, S., Zhang, G. and Schoeman, B. J., Thin Molecular Sieve Films on Noble Metal Substrates. Zeolites, 18, 387 (1997).

Suzuki, O., Adsorption and Fouling at Stainless Steel Surfaces. Encyclopedia of Surface and Colloid Science, Taylor and Francis, p. 185 (2006).

Tatlier, M., Tantekin-Ersolmaz, Ş. B. and ErdemŞenatalar, A., A Novel Approach to Enhance Heat and Mass Transfer in Adsorption Heat Pumps Using the Zeolite-Water Pair. Microporous and Mesoporous Materials, 27, 1 (1999).

Tatlier, M., Cigizoglu, K. B. and Erdem-Senatalar, A., Preparation of Zeolite Coatings on Large Metal Surfaces for Adsorption Heat Pump Applications. Proceedings of Heat SET, EditionsGRETh, France, p. 395 (2007).

Tatlier, M., Fractal Growth of Zeolite Coatings Prepared by the Substrate Heating Method. Fractals, 11, 77 (2003).

Tsay, C. S. and Chiang, A. S. T., Supported Zeolite Membrane by Vapor-Phase Regrowth. AIChE Journal, 46, 616 (2000).

Valtchev, V., Mintova, S. and Vasilev, I., The Effect of Plastic Deformation and Thermal Annealing of the Copper Substrate on the Zeolite Film Formation. Journal of the Chemical SocietyChemical Communications, 8, 979 (1994).

Valtchev, V., Hedlund, J., Schoeman, B. J., Sterte, J. and Mintova, S., Deposition of Continuous Silicalite-1 Films on Inorganic Fibers. Microporous Materials, 8, 93 (1997).

Yamazaki, S. and Tsutsumi, K., Synthesis of an AType Zeolite Membrane on Silicon-Oxide FilmSilicon, Quartz Plate and Quartz Fiber Filter. Microporous Materials, 4, 205 (1995).

Yuranov, I., Renken, A. and Kiwi-Minsker, L., Zeolite/Sintered Metal Fibers Composites as Effective Structured Catalysts. Applied Catalysis A, 281, 55 (2005). 